\title{
Geometric Properties of Normalized Wright Functions
}

\author{
Nizami Mustafa \\ Department of Mathematics, Faculty of Science and Letters, Kafkas University, Kars TR-36100, Turkey; \\ nizamimustafa@gmail.com; Tel.: +90-538-603-4878 \\ Academic Editor: Mehmet Pakdemirli \\ Received: 5 February 2016; Accepted: 28 April 2016; Published: 4 May 2016
}

\begin{abstract}
The purpose of the present paper is to investigate some characterizations for the Wright functions to be in the subclasses $S^{*}(\alpha, \beta)$ and $C(\alpha, \beta)(\alpha, \beta \in[0,1))$. Several sufficient conditions are obtained for the normalized Wright functions to be in these classes. Results obtained in this paper are new and their usefulness is put forth by several corollaries.
\end{abstract}

Keywords: Wright function; starlike function; convex function

\section{Introduction, Definitions and Preliminaries}

It is well known that special functions play an important role in geometric function theory. It is also well known that the application area of special functions is not limited to the theory of geometric functions. There are wide ranges of applications of these functions in many problems as well as in other branches of mathematics and applied sciences.

In this paper, we will examine the function

$$
\phi(\lambda, \mu ; z)=\sum_{n=0}^{\infty} \frac{1}{\Gamma(\lambda n+\mu)} \frac{z^{n}}{n !}, \lambda>-1, \mu, z \in \mathbb{C}
$$

named after the British Mathematician E. M. Wright. This function appeared for the first time in the case $\lambda>0$ in connection with Wright's investigations in the asymptotic theory of partitions [1]. Later on, many other applications were found in Mikusinski operational calculus and in the theory of integral transforms of the Hankel type. Furthermore, extending the methods of Lie groups in partial differential equations to the partial differential equations of fractional order, it was shown that some of the group-invariant solutions of these equations can be given in terms of the Wright and the generalized Wright functions. Recently, Wright functions have appeared in papers related to partial differential equations of fractional order. It was found that the corresponding Green functions can be represented in terms of the Wright functions [2,3]. A series of papers are devoted to the application of the Wright functions in partial differential equations of fractional order extending the classical diffusion and wave equations [4]. Mainardi obtained the result for a fractional diffusion wave equation in terms of the fractional Green function involving the Wright functions [5]. The scale-variant solutions of some partial differential equations of fractional order were obtained in terms of special cases of the generalized Wright functions by Buckwar and Luchko [6] and Luchko and Gorenflo [7].

If $\lambda$ is a positive rational number, then the Wright function $\phi(\lambda, \mu, z)$ can be represented in terms of the more familiar generalized hypergeometric functions (see [8], Section 2.1). In particular, when $\lambda=1$ and $\mu=p+1$, the functions $\phi\left(1, p+1 ;-z^{2} / 4\right)$ are expressed in terms of the Bessel functions $J_{p}(z)$, given as follows:

$$
J_{p}(z)=\left(\frac{z}{2}\right)^{p} \phi\left(1, p+1 ;-\frac{z^{2}}{4}\right)=\sum_{n=0}^{\infty} \frac{(-1)^{n}}{\Gamma(n+p+1)} \frac{(z / 2)^{2 n+p}}{n !}
$$


Furthermore, the function $\phi(\lambda, p+1 ;-z) \equiv J_{p}^{\lambda}(z)(\lambda>0, p>-1)$ is known as the generalized Bessel function (sometimes it is also called the Bessel-Wright function). Also, the Wright function generalizes various simple functions such as the Array function, the Whittaker function, (Wright-type) entire auxiliary functions, etc. For the details, we refer to [8].

Let $A$ be the class of analytic functions $f(z)$ in the open unit disk $U=\{z \in \mathbb{C}:|z|<1\}$, normalized by $f(0)=0=f^{\prime}(0)-1$ of the form

$$
f(z)=z+\sum_{n=2}^{\infty} a_{n} z^{n}
$$

Also, let $S^{*}(\alpha)$ and $C(\alpha)$ denote the subclasses of $A$ consisting of functions which are, respectively, starlike and convex of order $\alpha(\alpha \in[0,1))$ in the open unit disk $U$. Thus, we have $[9,10]$

$$
\begin{gathered}
S^{*}(\alpha)=\left\{f \in A: \operatorname{Re}\left(\frac{z f^{\prime}(z)}{f(z)}\right)>\alpha, z \in U\right\}, \alpha \in[0,1) \\
C(\alpha)=\left\{f \in A: \operatorname{Re}\left(1+\frac{z f^{\prime \prime}(z)}{f^{\prime}(z)}\right)>\alpha, z \in U\right\}, \alpha \in[0,1)
\end{gathered}
$$

In the special case, $S^{*}=S^{*}(0)$ and $C=C(0)$ are starlike and convex functions, respectively.

An interesting generalization of the functions, the classes $S^{*}(\alpha)$ and $C(\alpha)$ are provided by the classes $S^{*}(\alpha, \beta)$ and $C(\alpha, \beta)$ of functions $f \in A$, which satisfies the following conditions:

$$
\begin{gathered}
S^{*}(\alpha, \beta)=\left\{f \in A: \operatorname{Re}\left(\frac{z f^{\prime}(z)+\beta z^{2} f^{\prime \prime}(z)}{f(z)}\right)>\alpha, z \in U\right\}, \alpha, \beta \in[0,1) \\
C(\alpha, \beta)=\left\{f \in A: \operatorname{Re}\left(\frac{\left(z f^{\prime}(z)+\beta z^{2} f^{\prime \prime}(z)\right)^{\prime}}{f^{\prime}(z)}\right)>\alpha, z \in U\right\}, \alpha, \beta \in[0,1)
\end{gathered}
$$

respectively.

Clearly, for $\beta=0$, we have $S^{*}(\alpha, 0)=S^{*}(\alpha)$ and $C(\alpha, 0)=C(\alpha)$.

Remark 1.1. For $f \in A$, observe that $f(z) \in C(\alpha, \beta) \Leftrightarrow z f^{\prime}(z) \in S^{*}(\alpha, \beta)$.

Note that the Wright function $\phi(\lambda, \mu ; z)$ defined by Equation (1) does not belong to class $A$. Thus, it is natural to consider the following normalization of the Wright function:

$$
\Phi^{(1)}(\lambda, \mu ; z):=\Gamma(\mu) \mathrm{z} \phi(\lambda, \mu ; z)=\sum_{n=0}^{\infty} \frac{\Gamma(\mu)}{\Gamma(\lambda n+\mu)} \frac{z^{n+1}}{n !}, \lambda>-1, \mu>0, z \in U
$$

and

$$
\begin{gathered}
\Phi^{(2)}(\lambda, \mu ; z):=\Gamma(\lambda+\mu)\left(\phi(\lambda, \mu ; z)-\frac{1}{\Gamma(\mu)}\right)=\sum_{n=0}^{\infty} \frac{\Gamma(\lambda+\mu)}{\Gamma(\lambda n+\lambda+\mu)} \frac{z^{n+1}}{(n+1) !} \\
\lambda>-1, \lambda+\mu>0, z \in U .
\end{gathered}
$$

Easily, we can write

$$
\begin{gathered}
\Phi^{(1)}(\lambda, \mu ; z)=z+\sum_{n=2}^{\infty} \frac{\Gamma(\mu)}{\Gamma(\lambda(n-1)+\mu)} \frac{z^{n}}{(n-1) !}, \lambda>-1, \mu>0, z \in U \\
\Phi^{(2)}(\lambda, \mu ; z)=z+\sum_{n=2}^{\infty} \frac{\Gamma(\lambda+\mu)}{\Gamma(\lambda(n-1)+\lambda+\mu)} \frac{z^{n}}{n !}, \lambda>-1, \lambda+\mu>0, z \in U
\end{gathered}
$$

Furthermore, observe that $\Phi^{(1)}(\lambda, \mu ; z)$ and $\Phi^{(2)}(\lambda, \mu ; z)$ are satisfied in the following equations:

$$
\lambda z\left(\Phi^{(1)}(\lambda, \mu ; z)\right)^{\prime}=(\mu-1) \Phi^{(1)}(\lambda, \mu-1 ; z)+(\lambda-\mu+1) \Phi^{(1)}(\lambda, \mu ; z)
$$




$$
\begin{gathered}
\Phi^{(2)}(\lambda, \mu ; z)=z+\sum_{n=2}^{\infty} \frac{\Gamma(\lambda+\mu)}{\Gamma(\lambda(n-1)+\lambda+\mu)} \frac{z^{n}}{n !}, \lambda>-1, \lambda+\mu>0, z \in U \\
z\left(\Phi^{(2)}(\lambda, \mu ; z)\right)^{\prime}=\Phi^{(1)}(\lambda, \lambda+\mu ; z) \text { and } V_{\lambda, \mu}^{\prime}(z)=\frac{\Gamma(\mu)}{\Gamma(\lambda+\mu)} V_{\lambda, \lambda+\mu}(z)
\end{gathered}
$$

where $V_{\lambda, \mu}(z)=\frac{\Phi^{(1)}(\lambda, \mu ; z)}{z}$.

Also,

$$
-\Phi^{(1)}(1, p+1 ;-z)=\bar{J}_{p}(z):=\Gamma(p+1) z^{1-p / 2} J_{p}(2 \sqrt{z})
$$

Here, $\bar{J}_{p}(z)$ is the normalized Bessel function.

Several researchers have studied classes of analytic functions involving special function $F \subset A$ to find different conditions such that the members of $F$ have certain geometric properties such as starlikeness and convexity in $U$. There is extensive literature dealing with various properties, generalizations and applications of different types of hypergeometric functions [4,11-17].

Recently, there have been many studies about various properties of hypergeometric functions. On this matter, one can see the works [8,11-22]. In these studies, geometric properties such as starlikeness, convexity and univalency have usually been investigated. Furthermore, in the work by Dziok and Srivastava in $[18,19]$, the analytic function classes related with the generalized hypergeometric function were studied. For these classes, coefficient analysis, distortion theorems, extreme points and the radii of convexity and starlikeness are given. In [20], classes related to some generalized Fox-Wright hypergeometric functions and convolution operations were taken and their properties were researched. Moreover, in [21] it was shown that Dziok-Srivastava and Srivastava-Wright operators were defined from $A$ to $A$ and a criterion was given for these operators to be univalent. In [22], univalence, starlikeness and convexity of the following generalized hypergeometric function given in the formula below were investigated:

$$
{ }_{p} F_{q}(z):={ }_{p} F_{q}\left(a_{1}, \ldots, a_{p} ; b_{1}, \ldots, b_{q} ; z\right)=\sum_{n=0}^{\infty} \frac{\left(a_{1}\right)_{n} \cdots\left(a_{p}\right)_{n}}{\left(b_{1}\right)_{n} \cdots\left(b_{q}\right)_{n}} \frac{z^{n}}{n !}, p \leqslant q+1
$$

Furthermore, in this work, applications of generalized hypergeometric functions were given.

In the works mentioned above, various properties of generalized hypergeometric functions were investigated.

In the geometric function theory, investigating geometric properties of an analytic function given in the open unit disc is a research subject. On this subject, there are many works such as [11-13,17]. In [23], Prajapat investigated some geometric properties such as starlikeness and convexity of the normalized Wright functions $\Phi^{(1)}(\lambda, \mu ; z)$ and $\Phi^{(2)}(\lambda, \mu ; z)$. Investigating geometric properties of the normalized Wright functions $\Phi^{(1)}(\lambda, \mu ; z)$ and $\Phi^{(2)}(\lambda, \mu ; z)$ in a class more generalized than the class of starlike and convex functions is also a research interest. In this paper, we investigate the normalized Wright functions $\Phi^{(1)}(\lambda, \mu ; z)$ and $\Phi^{(2)}(\lambda, \mu ; z)$ in the classes of $S^{*}(\alpha, \beta)$ and $C(\alpha, \beta)$ which are generalizations of $S^{*}(\alpha)$ and $C(\alpha)$, respectively. Several sufficient conditions for the normalized Wright functions $\Phi^{(1)}(\lambda, \mu ; z)$ and $\Phi^{(2)}(\lambda, \mu ; z)$ to be in the classes of $S^{*}(\alpha, \beta)$ and $C(\alpha, \beta)$ are given. From the obtained results, using specific values for $\alpha$ and $\beta$ parameters, better results than those of previous results found in the literature are obtained.

The following lemmas will be required in our investigation.

Lemma 1.1. [24] A function $f \in A$ belongs to the class $S^{*}(\alpha, \beta)$ if

$$
\sum_{n=2}^{\infty}(n+\beta n(n-1)-\alpha)\left|a_{n}\right| \leqslant 1-\alpha .
$$


Lemma 1.2. [24] A function $f \in A$ belongs to the class $C(\alpha, \beta)$ if

$$
\sum_{n=2}^{\infty} n(n+\beta n(n-1)-\alpha)\left|a_{n}\right| \leqslant 1-\alpha
$$

2. Sufficient Conditions for the Wright Functions to be Classes $S^{*}(\alpha, \beta)$ and $C(\alpha, \beta)$

In this section, we will give sufficient conditions for the normalized Wright functions to be in the classes $S^{*}(\alpha, \beta)$ and $C(\alpha, \beta)$.

Theorem 2.1. Let $\lambda \geqslant 1$ and assume the following condition is satisfied:

$$
(1-\alpha)(\mu+1)\left[\left(2-e^{1 /(\mu+1)}\right) \mu+\left(1-e^{1 /(\mu+1)}\right)\right]-(1+2 \beta)(\mu+1) e^{1 /(\mu+1)}-\beta e^{1 /(\mu+1)} \geqslant 0
$$

Then, the normalized Wright function $\Phi^{(1)}(\lambda, \mu ; z)$ belongs to the class $S^{*}(\alpha, \beta)$.

Proof. Since

$$
\Phi^{(1)}(\lambda, \mu ; z)=\mathrm{z}+\sum_{n=2}^{\infty} \frac{\Gamma(\mu)}{\Gamma((n-1) \lambda+\mu)} \frac{z^{n}}{(n-1) !}
$$

by virtue of Lemma 1.1, it suffices to show that

$$
\sum_{n=2}^{\infty}[n+\beta n(n-1)-\alpha] \frac{\Gamma(\mu)}{\Gamma((n-1) \lambda+\mu)} \frac{1}{(n-1) !} \leqslant 1-\alpha
$$

Let

$$
L_{1}(\lambda, \mu ; \alpha, \beta)=\sum_{n=2}^{\infty}\left[n^{2} \beta+n(1-\beta)-\alpha\right] \frac{\Gamma(\mu)}{\Gamma((n-1) \lambda+\mu)} \frac{1}{(n-1) !} .
$$

Setting $n^{2}=(n-1)(n-2)+3(n-1)+1, n=(n-1)+1$ and by simple computation, we get

$$
\begin{aligned}
L_{1}(\lambda, \mu ; \alpha, \beta)= & \sum_{n=3}^{\infty} \frac{\beta}{(n-3) !} \frac{\Gamma(\mu)}{\Gamma((n-1) \lambda+\mu)}+\sum_{n=2}^{\infty} \frac{1+2 \beta}{(n-2) !} \frac{\Gamma(\mu)}{\Gamma((n-1) \lambda+\mu)} \\
& +\sum_{n=2}^{\infty} \frac{1-\alpha}{(n-1) !} \frac{\Gamma(\mu)}{\Gamma((n-1) \lambda+\mu)} .
\end{aligned}
$$

Under the hypothesis $\lambda \geqslant 1$, the inequality $\Gamma(n-1+\mu) \leqslant \Gamma((n-1) \lambda+\mu)$, for $n \in \mathbb{C}$, holds. This inequality is equivalent to

$$
\frac{\Gamma(\mu)}{\Gamma((n-1) \lambda+\mu)} \leqslant \frac{1}{(\mu)_{n-1}}, n \in \mathbb{C}
$$

Here, $(\mu)_{n}=\frac{\Gamma(n+\mu)}{\Gamma(\mu)}=\mu(\mu+1)(\mu+2) \cdots(\mu+n-1),(\mu)_{0}=1$ is the Pochhammer (or Appell) symbol defined in terms of the Euler gamma function. Using Equation (14), we obtain

$$
L_{1}(\lambda, \mu ; \alpha, \beta) \leqslant \sum_{n=3}^{\infty} \frac{\beta}{(n-3) !} \frac{1}{(\mu)_{n-1}}+\sum_{n=2}^{\infty} \frac{1+2 \beta}{(n-2) !} \frac{1}{(\mu)_{n-1}}+\sum_{n=2}^{\infty} \frac{1-\alpha}{(n-1) !} \frac{1}{(\mu)_{n-1}}
$$

Further, the inequality

$$
(\mu)_{n-1}=\mu(\mu+1)(\mu+2) \cdots(\mu+n-2) \geqslant \mu(\mu+1)^{n-2}, n \in \mathbb{C}
$$


holds and this is equivalent to $1 /(\mu)_{n-1} \leqslant 1 / \mu(\mu+1)^{n-2}, n \in \mathbb{C}$. Using Equation (15), we obtain

$$
\begin{aligned}
L_{1}(\lambda, \mu ; \alpha, \beta) & \leqslant \sum_{n=3}^{\infty} \frac{\beta}{(n-3) !} \frac{1}{\mu(\mu+1)^{n-2}}+\sum_{n=2}^{\infty} \frac{1+2 \beta}{(n-2) !} \frac{1}{\mu(\mu+1)^{n-2}}+\sum_{n=2}^{\infty} \frac{1-\alpha}{(n-1) !} \frac{1}{\mu(\mu+1)^{n-2}} \\
& =\frac{\beta}{\mu(\mu+1)} e^{1 /(\mu+1)}+\frac{1+2 \beta}{\mu} e^{1 /(\mu+1)}+(1-\alpha) \frac{\mu+1}{\mu}\left(e^{1 /(\mu+1)}-1\right) \leqslant 1-\alpha .
\end{aligned}
$$

Hence,

$$
(1-\alpha)\left[\left(2-e^{1 /(\mu+1)}\right)+\frac{1}{\mu}\left(1-e^{1 /(\mu+1)}\right)\right]-\frac{\beta}{\mu(\mu+1)} e^{1 /(\mu+1)}-\frac{1+2 \beta}{\mu} e^{1 /(\mu+1)} \geqslant 0 .
$$

This completes the proof of Theorem 2.1.

Theorem 2.2. Let $\lambda \geqslant 1$ and assume the following condition is satisfied:

$$
\begin{gathered}
(1-\alpha)\left[\left(2-e^{1 /(\mu+1)}\right) \mu+\left(1-e^{1 /(\mu+1)}\right)\right](\mu+1)^{2} \\
-\left[(3+4 \beta-\alpha)(\mu+1)^{2}+(1+5 \beta)(\mu+1)+\beta\right] e^{1 /(\mu+1)} \geqslant 0 .
\end{gathered}
$$

Then, normalized Wright function $\Phi^{(1)}(\lambda, \mu ; z)$ belongs to the class $C(\alpha, \beta)$.

Proof. Since

$$
\Phi^{(1)}(\lambda, \mu ; z)=z+\sum_{n=2}^{\infty} \frac{\Gamma(\mu)}{\Gamma((n-1) \lambda+\mu)} \frac{z^{n}}{(n-1) !}
$$

and by virtue of Lemma 1.2, it suffices to show that

$$
\sum_{n=2}^{\infty} n[n+\beta n(n-1)-\alpha] \frac{\Gamma(\mu)}{\Gamma((n-1) \lambda+\mu)} \frac{1}{(n-1) !} \leqslant 1-\alpha .
$$

Let

$$
L_{2}(\lambda, \mu ; \alpha, \beta)=\sum_{n=2}^{\infty}\left[n^{3} \beta+n^{2}(1-\beta)-n \alpha\right] \frac{\Gamma(\mu)}{\Gamma((n-1) \lambda+\mu)} \frac{1}{(n-1) !} .
$$

Setting

$$
\begin{aligned}
n^{3} \beta+n^{2}(1-\beta)-n \alpha= & \beta(n-1)(n-2)(n-3)+(1+5 \beta)(n-1)(n-2) \\
& +(3+4 \beta-\alpha)(n-1)+(1-\alpha)
\end{aligned}
$$

and by simple computation, we can write

$$
\begin{aligned}
L_{2}(\lambda, \mu ; \alpha, \beta)= & \sum_{n=4}^{\infty} \frac{\beta}{(n-4) !} \frac{\Gamma(\mu)}{\Gamma((n-1) \lambda+\mu)}+\sum_{n=3}^{\infty} \frac{1+5 \beta}{(n-3) !} \frac{\Gamma(\mu)}{\Gamma((n-1) \lambda+\mu)} \\
& +\sum_{n=2}^{\infty} \frac{3+4 \beta-\alpha}{(n-2) !} \frac{\Gamma(\mu)}{\Gamma((n-1) \lambda+\mu)}+\sum_{n=2}^{\infty} \frac{1-\alpha}{(n-1) !} \frac{\Gamma(\mu)}{\Gamma((n-1) \lambda+\mu)} .
\end{aligned}
$$


Using Equations (14) and (15), we obtain

$$
\begin{aligned}
L_{2}(\lambda, \mu ; \alpha, \beta) \leqslant & \sum_{n=4}^{\infty} \frac{\beta}{(n-4) !} \frac{1}{\mu(\mu+1)^{n-2}}+\sum_{n=3}^{\infty} \frac{1+5 \beta}{(n-3) !} \frac{1}{\mu(\mu+1)^{n-2}} \\
& +\sum_{n=2}^{\infty} \frac{3+4 \beta-\alpha}{(n-2) !} \frac{1}{\mu(\mu+1)^{n-2}}+\sum_{n=2}^{\infty} \frac{1-\alpha}{(n-1) !} \frac{1}{\mu(\mu+1)^{n-2}}=\frac{\beta}{\mu(\mu+1)^{2}} e^{1 /(\mu+1)} \\
& +\frac{1+5 \beta}{\mu(\mu+1)} e^{1 /(\mu+1)}+\frac{3+4 \beta-\alpha}{\mu} e^{1 /(\mu+1)}+(1-\alpha) \frac{\mu+1}{\mu}\left(e^{1 /(\mu+1)}-1\right) .
\end{aligned}
$$

Thus,

$$
\begin{aligned}
L_{2}(\lambda, \mu ; \alpha, \beta) \leqslant & \frac{\beta}{\mu(\mu+1)^{2}} e^{1 /(\mu+1)}+\frac{1+5 \beta}{\mu(\mu+1)} e^{1 /(\mu+1)}+\frac{3+4 \beta-\alpha}{\mu} e^{1 /(\mu+1)} \\
& +(1-\alpha)\left[\left(e^{1 /(\mu+1)}-1\right)+\frac{1}{\mu}\left(e^{1 /(\mu+1)}-1\right)\right] \leqslant 1-\alpha,
\end{aligned}
$$

which is equivalent to

$$
\begin{array}{r}
(1-\alpha)\left[\left(2-e^{1 /(\mu+1)}\right)+\frac{1}{\mu}\left(1-e^{1 /(\mu+1)}\right)\right]-\frac{\beta}{\mu(\mu+1)^{2}} e^{1 /(\mu+1)} \\
-\frac{1+5 \beta}{\mu(\mu+1)} e^{1 /(\mu+1)}-\frac{3+4 \beta-\alpha}{\mu} e^{1 /(\mu+1)} \geqslant 0 .
\end{array}
$$

This evidently completes the proof of Theorem 2.2.

By setting $\beta=0$ in Theorems 2.1 and 2.2, we arrive at the following corollaries.

Corollary 2.1. The normalized Wright function $\Phi^{(1)}(\lambda, \mu ; z)$ belongs to the class $S^{*}(\alpha)$ if $\lambda \geqslant 1$ and the following condition is satisfied:

$$
(1-\alpha)\left[\left(2-e^{1 /(\mu+1)}\right) \mu+\left(1-e^{1 /(\mu+1)}\right)\right]-e^{1 /(\mu+1)} \geqslant 0 .
$$

Corollary 2.2. The normalized Wright function $\Phi^{(1)}(\lambda, \mu ; z)$ belongs to the class $C(\alpha)$ if $\lambda \geqslant 1$ and the following condition is satisfied:

$$
(1-\alpha)\left[\left(2-e^{1 /(\mu+1)}\right) \mu+\left(1-e^{1 /(\mu+1)}\right)\right](\mu+1)-[(3-\alpha)(\mu+1)+1] e^{1 /(\mu+1)} \geqslant 0 .
$$

By taking $\alpha=0$ in Corollary 2.1 and Corollary 2.2, we obtain the following corollaries.

Corollary 2.3. Let $\lambda \geqslant 1$ and $\mu>x_{0}$, where $x_{0} \cong 2.4898$ is the root of the equation

$$
2 x-(x+2) e^{1 /(x+1)}+1=0,
$$

then $\Phi^{(1)} \in S^{*}$.

Corollary 2.4. Let $\lambda \geqslant 1$ and $\mu>x_{1}$, where $x_{1} \cong 4.8523$ is the root of the equation

$$
\left(2-e^{1 /(x+1)}\right) x^{2}+\left(3-5 e^{1 /(x+1)}\right) x-5 e^{1 /(x+1)}+1=0,
$$

then $\Phi^{(1)} \in C$.

Theorem 2.3. Let $\lambda \geqslant 1$ and assume the following condition is satisfied:

$$
(1-\alpha)\left[2(\lambda+\mu)+1-\left(e^{1 /(\lambda+\mu+1)}-1\right)(\lambda+\mu+1)^{2}\right]-(\lambda+\mu+1)\left(e^{1 /(\lambda+\mu+1)}-1\right)-\beta e^{1 /(\lambda+\mu+1)} \geqslant 0 .
$$

Then, normalized Wright function $\Phi^{(2)}(\lambda, \mu ; z)$ belongs to the class $S^{*}(\alpha, \beta)$. 
Proof. Since

$$
\Phi^{(2)}(\lambda, \mu ; z)=z+\sum_{n=2}^{\infty} \frac{\Gamma(\lambda+\mu)}{\Gamma(\lambda(n-1)+\lambda+\mu)} \frac{z^{n}}{n !}
$$

and by virtue of Lemma 1.1, it suffices to show that

$$
\sum_{n=2}^{\infty}[n+\beta n(n-1)-\alpha] \frac{\Gamma(\lambda+\mu)}{\Gamma((n-1) \lambda+\lambda+\mu)} \frac{1}{n !} \leqslant 1-\alpha .
$$

Let

$$
L_{3}(\lambda, \mu ; \alpha, \beta)=\sum_{n=2}^{\infty}[n+\beta n(n-1)-\alpha] \frac{\Gamma(\lambda+\mu)}{\Gamma((n-1) \lambda+\lambda+\mu)} \frac{1}{n !} .
$$

By simple computation, we obtain

$$
\begin{gathered}
L_{3}(\lambda, \mu ; \alpha, \beta)=\sum_{n=2}^{\infty} \frac{\beta}{(n-2) !} \frac{\Gamma(\lambda+\mu)}{\Gamma(\lambda(n-1)+\lambda+\mu)}+\sum_{n=2}^{\infty}\left(1-\frac{1}{n}\right) \frac{1}{(n-1) !} \frac{\Gamma(\lambda+\mu)}{\Gamma(\lambda(n-1)+\lambda+\mu)} \\
+\sum_{n=2}^{\infty} \frac{1-\alpha}{n !} \frac{\Gamma(\lambda+\mu)}{\Gamma(\lambda(n-1)+\lambda+\mu)} .
\end{gathered}
$$

Using Equations (14) and (15), with $\mu \equiv \lambda+\mu$, we get

$$
\begin{aligned}
L_{3}(\lambda, \mu ; \alpha, \beta) \leqslant & \sum_{n=2}^{\infty} \frac{\beta}{(n-2) !} \frac{1}{(\lambda+\mu)(\lambda+\mu+1)^{n-2}}+\sum_{n=2}^{\infty} \frac{1}{(n-1) !} \frac{1}{(\lambda+\mu)(\lambda+\mu+1)^{n-2}} \\
& +\sum_{n=2}^{\infty} \frac{1-\alpha}{n !} \frac{1}{(\lambda+\mu)(\lambda+\mu+1)^{n-2}}=\frac{\beta}{\lambda+\mu} e^{1 /(\lambda+\mu+1)}+\frac{\lambda+\mu+1}{\lambda+\mu}\left(e^{1 /(\lambda+\mu+1)}-1\right) \\
& +\frac{(1-\alpha)(\lambda+\mu+1)^{2}}{\lambda+\mu}\left(e^{1 /(\lambda+\mu+1)}-\frac{1}{\lambda+\mu+1}-1\right) .
\end{aligned}
$$

Thus,

$$
\begin{aligned}
L_{3}(\lambda, \mu ; \alpha, \beta) \leqslant & \frac{\beta}{\lambda+\mu} e^{1 /(\lambda+\mu+1)}+\frac{\lambda+\mu+1}{\lambda+\mu}\left(e^{1 /(\lambda+\mu+1)}-1\right) \\
& +\frac{(1-\alpha)(\lambda+\mu+1)^{2}}{\lambda+\mu}\left(e^{1 /(\lambda+\mu+1)}-\frac{1}{\lambda+\mu+1}-1\right) \leqslant 1-\alpha
\end{aligned}
$$

which is equivalent to

$$
\begin{array}{r}
(1-\alpha)(\lambda+\mu)-(1-\alpha)\left(e^{1 /(\lambda+\mu+1)}-1\right)(\lambda+\mu+1)^{2}+(1-\alpha)(\lambda+\mu+1) \\
-(\lambda+\mu+1)\left(e^{1 /(\lambda+\mu+1)}-1\right)-\beta e^{1 /(\lambda+\mu+1)} \geqslant 0 .
\end{array}
$$

Thus, the proof of Theorem 2.3 is complete.

By setting $\beta=0$ in Theorem 2.3, we arrive at the following corollary.

Corollary 2.5. The normalized Wright function $\Phi^{(2)}(\lambda, \mu ; z)$ belongs to the class $S^{*}(\alpha)$ if $\lambda \geqslant 1$ and the following condition is satisfied:

$$
(1-\alpha)\left[2(\lambda+\mu)+1-\left(e^{1 /(\lambda+\mu+1)}-1\right)(\lambda+\mu+1)^{2}\right]-(\lambda+\mu+1)\left(e^{1 /(\lambda+\mu+1)}-1\right) \geqslant 0 .
$$

By taking $\alpha=0$ in Corollary 2.5, we obtain the following corollary.

Corollary 2.6. Let $\lambda \geqslant 1$ and $\lambda+\mu>x_{2}$, where $x_{2} \cong 1.7703$ is the root of the equation

$$
2 x-(x+1)(x+2)\left(e^{1 /(x+1)}-1\right)+1=0,
$$


then $\Phi^{(2)} \in S^{*}$.

Theorem 2.4. Let $\lambda \geqslant 1$ and assume the following condition is satisfied:

$$
\begin{array}{r}
(1-\alpha)(\lambda+\mu+1)\left[\left(2-e^{1 /(\lambda+\mu+1)}\right)(\lambda+\mu)+\left(1-e^{1 /(\lambda+\mu+1)}\right)\right] \\
-[(1+2 \beta)(\lambda+\mu+1)+\beta] e^{1 /(\lambda+\mu+1)} \geqslant 0,
\end{array}
$$

then normalized Wright function $\Phi^{(2)}(\lambda, \mu ; z)$ belongs to the class $C(\alpha, \beta)$.

Proof. The function $\Phi^{(2)}(\lambda, \mu ; z)$ belongs to the class $C(\alpha, \beta)$ if and only if $z \cdot\left(\Phi^{(2)}(\lambda, \mu ; z)\right)^{\prime}$ is in the class $S^{*}(\alpha, \beta)$, but from Equation (11) $z \cdot\left(\Phi^{(2)}(\lambda, \mu ; z)\right)^{\prime}=\Phi^{(1)}(\lambda, \lambda+\mu ; z)$. Then, using Theorem 2.1, we complete the proof of this theorem.

By setting $\beta=0$ in Theorem 2.4, we arrive at the following corollary.

Corollary 2.7. The normalized Wright function $\Phi^{(2)}(\lambda, \mu ; z)$ belongs to the class $C(\alpha)$ if $\lambda \geqslant 1$ and the following condition is satisfied:

$$
(1-\alpha)\left[\left(2-e^{1 /(\lambda+\mu+1)}\right)(\lambda+\mu)+\left(1-e^{1 /(\lambda+\mu+1)}\right)\right]-e^{1 /(\lambda+\mu+1)} \geqslant 0 .
$$

By taking $\alpha=0$ in Corollary 2.7, we obtain the following corollary.

Corollary 2.8. Let $\lambda \geqslant 1$ and $\lambda+\mu>x_{0}$, where $x_{0} \cong 2.4898$ is the root of the equation

$$
2 x-(x+2) e^{1 /(x+1)}+1=0,
$$

then $\Phi^{(2)} \in C$.

Remark 2.1. Note that the results obtained in Corollary 2.3 and Corollary 2.8 are better versions of the results given by Prajapat (see [23], (p. 206, Theorem 2.7 (a), (c))).

\section{Sufficient Conditions for the Normalized Bessel Function $\bar{J}_{p}(z)$}

In this section, we will give sufficient conditions for the normalized Bessel function to be in the classes $S^{*}(\alpha, \beta)$ and $C(\alpha, \beta)$.

Setting $\lambda=1, \mu=p+1$ and $z=-z$ in Theorem 2.1, from Equation (13) we obtain the following results.

Theorem 3.1. The normalized Bessel function $\bar{J}_{p}(z)$ belongs to the class $S^{*}(\alpha, \beta)$ if

$$
(1-\alpha)(p+2)\left[\left(2-e^{1 /(p+2)}\right)(p+1)+\left(1-e^{1 /(p+2)}\right)\right]-[(1+2 \beta)(p+2)+\beta] e^{1 /(p+2)} \geqslant 0
$$

Corollary 3.1. The normalized Bessel function $\bar{J}_{p}(z)$ belongs to the class $S^{*}(\alpha)$ if

$$
(1-\alpha)\left[\left(2-e^{1 /(p+2)}\right)(p+1)+\left(1-e^{1 /(p+2)}\right)\right]-e^{1 /(p+2)} \geqslant 0
$$

Corollary 3.2. The normalized Bessel function $\bar{J}_{p}(z)$ belongs to the class $S^{*}$ if $p>x_{0}-1$, where $x_{0} \cong 2.4898$ is the root of the equation

$$
2 x-(2+x) e^{1 /(x+1)}+1=0 .
$$

Setting $\lambda=1, \mu=p+1$ and $z=-z$ in Theorem 2.2, from Equation (13) we obtain the following results. 
Theorem 3.2. The normalized Bessel function $\bar{J}_{p}(z)$ belongs to the class $C(\alpha, \beta)$ if

$$
\begin{aligned}
& (1-\alpha)(p+2)^{2}\left[\left(2-e^{1 /(p+2)}\right)(p+1)+\left(1-e^{1 /(p+2)}\right)\right] \\
& -\left[(3+4 \beta-\alpha)(p+2)^{2}+(1+5 \beta)(p+2)+\beta\right] e^{1 /(p+2)} \geqslant 0
\end{aligned}
$$

Corollary 3.3. The normalized Bessel function $\bar{J}_{p}(z)$ belongs to the class $C(\alpha)$ if

$$
(1-\alpha)(p+2)\left[\left(2-e^{1 /(p+2)}\right)(p+1)+\left(1-e^{1 /(p+2)}\right)\right]-[(3-\alpha)(p+2)+1] e^{1 /(p+2)} \geqslant 0 .
$$

Corollary 3.4. The normalized Bessel function $\bar{J}_{p}(z)$ belongs to the class $C$ if $p>x_{1}-1$, where $x_{1} \cong 4.8523$ is the root of the equation

$$
\left(2-e^{1 /(x+1)}\right) x^{2}+\left(3-5 e^{1 /(x+1)}\right) x-5 e^{1 /(x+1)}+1=0 .
$$

\section{Concluding Remarks}

In this study, two kinds of normalizations of the Wright function are considered. These normalized Wright functions are examined in the generalized classes of the starlike and convex function classes. In the study, several sufficient conditions for the normalized Wright functions are obtained. The results obtained in this study are compared with the results obtained by Prajapat [23].

Acknowledgments: I would like to express my gratitude to the anonymous reviewers for the careful reading of the paper and for their invaluable comments that helped me to improve it considerably.

Conflicts of Interest: The author declares that there is no conflict of interests regarding the publication of this paper.

\section{References}

1. Wright, E.M. On the coefficients of power series having exponential singularities. J. Lond. Math. Soc. 1933, 8, 71-79. [CrossRef]

2. Podlubny, I. Fractional Differential Equations; Academic Press: San Diego, CA, USA, 1999.

3. Samko, S.G.; Kilbas, A.A.; Marichev, O.I. Fractional Integrals and Derivatives: Theory and Applications; Gordon and Breach: New York, NY, USA, 1993.

4. Kilbas, A.A.; Srivastava, H.M.; Trujillo, J.J. Theory and applications of fractional differential equations. In North-Holland Mathematical Studies; Elsevier (North-Holland) Science Publishers: Amsterdam, The Netherlands, 2006; Volume 204.

5. Mainardi, F. Fractional Calculus: Some Basic Problems in Continuum and Statistical Mechanics; Springer Verlag: Wien, Austria, 1971.

6. Buckwar, E.; Luchko, Y. Invariance of a partial differential equation of fractional order under the Lie group of scaling transformations. J. Math. Anal. Appl. 1998, 227, 81-97. [CrossRef]

7. Luchko, Y.; Gorenflo, R. Scale-invariant solutions of a partial differential equation of fractional order. Fract. Calc. Appl. Anal. 1998, 1, 63-78.

8. Gorenflo, R.; Luchko, Y.; Mainardi, F. Analytic properties and applications of Wright functions. Fract. Calc. Appl. Anal. 1999, 2, 383-414.

9. Duren, P.L. Univalent Functions, Grundlehren der Mathematischen Wissenshaften, Bd. 259; Springer-Verlag: New York, NY, USA, 1983.

10. Current Topics in Analytic Function Theory; Srivastava, H.M., Owa, S., Eds.; World Scientific Publishing Company: Singapore, 1992.

11. Baricz, A.; Ponnusamy, S. Starlikeness and convexity of generalized Bessel functions. Integral Transforms Spec. Funct. 2010, 21, 641-653. [CrossRef]

12. Miller, S.S.; Mocanu, P.T. Univalence of Gaussian and confluent hypergeometric functions. Proc. Am. Math. Soc. 1990, 110, 333-342. [CrossRef] 
13. Ponnusamy, S.; Ronning, F. Geometric properties for convolutions of hypergeometric functions and functions with the derivative in a half plane. Integral Transforms Spec. Funct. 1999, 8, 121-138. [CrossRef]

14. Ponnusamy, S.; Singh, V.; Vasundhra, P. Starlikeness and convexity of an integral transform. Integral Transforms Spec. Funct. 2004, 15, 267-280. [CrossRef]

15. Ponnusamy, S.; Vuorinen, M. Univalence and convexity properties for Gaussian hypergeometric functions. Rocky Mountain J. Math. 2001, 31, 327-353. [CrossRef]

16. Ponnusamy, S.; Vuorinen, M. Univalence and convexity properties for confluent hypergeometric functions. Complex Var. Elliptic Equ. 1998, 36, 73-97. [CrossRef]

17. Yagmur, N.; Orhan, H. Hardy space of generalized Struve functions. Complex Var. Elliptic Equ. 2014, 59, 929-936. [CrossRef]

18. Dziok, J.; Srivastava, H.M. Classes of analytic functions associated with the generalized hypergeometric function. Appl. Math. Comput. 1999, 103, 1-13. [CrossRef]

19. Dziok, J.; Srivastava, H.M. Certain subclasses of analytic functions associated with the generalized hypergeometric function. Integral Transforms Spec. Funct. 2003, 14, 7-18. [CrossRef]

20. Srivastava, H.M. Some Fox-Wright generalized hypergeometric functions and associated families of convolution operators. Appl. Anal. Discrete Math. 2007, 1, 56-71.

21. Kiryakova, V. Criteria for univalence of the Dziok-Srivastava and the Srivastava-Wright operators in the class A. Appl. Math. Comput. 2011, 218, 883-892. [CrossRef]

22. Owa, S.; Srivastava, H.M. Univalent and starlike generalized hypergeometric functions. Can. J. Math. 1987, 39, 1057-1077. [CrossRef]

23. Prajapat, J.K. Certain geometric properties of the Wright function. Integral Transforms Spec. Funct. 2015, 26, 203-212. [CrossRef]

24. Thulasiram, T.; Suchithra, K.; Sudharsan, T.V.; Murugusundaramoorthy, G. Some inclusion results associated with certain subclass of analytic functions involving Hohlov operator. Rev. R. Acad. Cienc. Exactas Fis. Nat. Ser.-A Math. 2014, 108, 711-720. [CrossRef]

(C) 2016 by the author; licensee MDPI, Basel, Switzerland. This article is an open access article distributed under the terms and conditions of the Creative Commons Attribution (CC-BY) license (http://creativecommons.org/licenses/by/4.0/). 